\title{
Alterations of the Beneficial Effect of Deep Inspiration in Scleroderma: Relationships between Lung Function and Imaging
}

\author{
Nicola Scichilone ${ }^{a}$ d Simona Reitano $^{a}$ Maria Bellia $^{b}$ Maria Riilic Alkis Togias $^{d}$ \\ Massimo Midiri ${ }^{b}$ Giovanni Triolo ${ }^{c}$ Vincenzo Bellia ${ }^{a}$ \\ a Dipartimento di Medicina, Pneumologia, Fisiologia e Nutrizione Umana, b Dipartimento di Biotecnologie e \\ Medicina Legale and ' Servizio di Reumatologia, Università di Palermo, Palermo, Italy; ${ }^{\mathrm{d}}$ Division of Allergy and \\ Clinical Immunology and Division of Respiratory and Critical Care Medicine, Department of Medicine, \\ School of Medicine, Johns Hopkins University, Baltimore, Md., USA
}

\section{Key Words}

Interstitial lung disease $\cdot$ Bronchial reactivity $\cdot$ Pulmonary mechanics

\begin{abstract}
Background: It has been postulated that the beneficial effects of deep inspiration are dependent on the magnitude of airway distension by virtue of airway to parenchyma interdependence. Objective: This study was designed to examine whether the changes that occur in pulmonary fibrosis affect the beneficial effect of deep inspiration. Methods: Thirty-one subjects with scleroderma underwent lung volume and diffusion capacity assessment as well as highresolution computed tomography. To assess the effect of deep inspiration, each subject underwent methacholine provocations in the absence of deep breaths. When the targeted change in lung function was achieved, subjects were asked to take 4 consecutive deep inspirations after which lung function measurements were repeated. The inspiratory vital capacity (IVC) was used as primary outcome. Results: Only 19 subjects attained the desired reduction in IVC in the absence of deep breaths. In those subjects, deep inspiration reversed the effect of methacholine on IVC by $48 \pm 8.4 \%$. The effect of deep inspiration correlated with DLCO\% pre-
\end{abstract}

dicted $(r=0.55, p=0.01)$, but not with high-resolution computed tomography abnormalities or baseline lung function measurements. When all subjects were considered, responsiveness to methacholine in the absence of deep inspiration was progressively lower with decreasing DLCO\% predicted values $(r=0.51, p=0.003)$. Conclusions: In systemic scleroderma, pulmonary abnormalities resulting in mild to moderate reduction in the diffusion capacity are associated with reduced ability of deep inspiration to reverse methacholineinduced early airway closure and bronchoconstriction. When diffusion capacity is severely affected, methacholine fails to produce obstructive airway changes.

Copyright $\odot 2008$ S. Karger AG, Basel

\section{Introduction}

The ability of deep inspirations to reverse bronchoconstriction that is experimentally induced in subjects free of respiratory disease is a well-known phenomenon [1-7]. A decrease in this beneficial effect of lung inflation has been documented in asthmatics $[1,4-7]$ and in individuals with chronic obstructive pulmonary disease (COPD) $[2,8,9]$. These observations have raised the hypothesis that impairment of this physiologic airway func-

\section{KARGER}

Fax +41613061234 E-Mail karger@karger.ch www.karger.com
(C) 2008 S. Karger AG, Basel

0025-7931/08/0763-0303\$24.50/0

Accessible online at:

www.karger.com/res
Nicola Scichilone

Dipartimento di Medicina, Pneumologia, Fisiologia e Nutrizione Umana

Università di Palermo, via Trabucco 180

IT-90146 Palermo (Italy)

Tel. +39 091680 2766, Fax +39 091689 1857, E-Mail n.scichilone@libero.it 
tion is one of the factors that contribute to the persistence of airway narrowing and early airway closure in chronic obstructive diseases [7].

The precise mechanism through which deep inspirations exert their beneficial effect and the causes of its impairment have not been elucidated. It has been postulated that the effect of deep inspirations is dependent on the magnitude of airway distension [10]. High-resolution computed tomography (HRCT) has demonstrated that lung inflation to total lung capacity distends the airways of healthy subjects and mild asthmatics to virtually the same extent [10], but that deep inspirations are less effective in distending the airways of more severe asthmatics [11]. In agreement with this observation, the reversibility of airway obstruction by deep inspiration, as measured by spirometry, is only minimally impaired in mild asthmatics, but is lost in those with more severe disease [11, 12 . It is possible that the impairment of the beneficial effect of deep inspiration reflects a reduction in airway-parenchyma interdependence, which is closely related to elastic recoil. This hypothesis is supported by our observation that the impairment of the effect of deep inspiration in subjects with COPD is related to the loss of alveolar septa [9]. Given that aging is characterized by a reduction in the connective tissue that surrounds the airways [13], the airway-parenchyma interdependence hypothesis is also supported by the observation that elderly individuals have reduced ability to reverse methacholine-induced bronchoconstriction with deep inspirations [14].

From the above observations, it is reasonable to infer that any factor that affects the interdependence between the intraparenchymal airways and the surrounding parenchyma influences the effectiveness of deep inspiration. We, therefore, hypothesized that the beneficial effect of deep inspirations would be enhanced in a condition in which lung elastic recoil is increased, such as that of lung fibrosis. We conducted this study to test this hypothesis. We selected the model of systemic sclerosis (SSc), a multisystem connective tissue disorder in which fibrotic involvement of the lung is a frequent and serious complication.

\section{Material and Methods}

\section{Subjects}

Subjects who fulfilled the diagnostic criteria of the American College of Rheumatology for SSc [15] participated in the study. They were recruited from the Unit of Rheumatology of the University Hospital of Palermo. After a complete clinical and serological evaluation, each subject was referred to our department for potential participation in this protocol. None of the subjects reported a diagnosis of COPD or other lung disease. One subject with history of asthma was excluded from the study. Known history of allergies or professional exposure to organic or inorganic dusts were also exclusion criteria. A total of 31 subjects were included in the study. None of them was a smoker. Current treatment consisted of iloprost (24 subjects) and calcium channel blockers ( 26 subjects); 5 subjects had not undergone any treatment by the time of the study. No subject was receiving any inhaled medication that could interfere with the study nor was any upper or lower respiratory infection reported during at least 4 weeks before evaluation. The study was approved by the local Ethics Committee and written informed consent was obtained from each participant.

\section{Study Design}

Before participating in the study, each subject underwent a complete clinical evaluation to assess the extent of skin and internal organ involvement, as well as a serologic evaluation to detect the presence of antinuclear antibody and anti-extractable nuclear antigen antibodies, including anti-Scl70 and anti-Sm. Transthoracic Doppler echocardiography was used as a noninvasive screening test to estimate pulmonary arterial pressure, as part of routine functional and hemodynamic assessments. On the basis of the established criteria of LeRoy et al. [16], subjects were classified into 2 groups, limited (ISSc) or diffuse (dSSc) SSc.

The study was divided into 2 phases. The first phase included baseline lung function and HRCT. The second phase aimed at assessing the effect of deep inspirations, as described in our previous work [7].

\section{Lung Volume and Diffusion Capacity}

We obtained lung volume measurements with the helium-dilution technique and a 10 -second single-breath test of diffusing capacity for carbon monoxide. Total lung capacity (TLC) and the pulmonary carbon monoxide diffusion capacity (DLCO) were determined; the results of these assessments were expressed as percentages of predicted values. A fully computerized watersealed Stead-Wells spirometer (Baires System; Biomedin, Padua, Italy) was used in the study. The spirometer is equipped with software that allows compliance with start and end of test American Thoracic Society criteria, which are confirmed on-line.

\section{Lung Imaging}

After completing the functional evaluation, each subject was referred for the HRCT session, which was performed within 1 week. The HRCT scans of the chest were carried out with scanner settings of $120 \mathrm{KVp}$ and $170 \mathrm{mAs}$, with a slice thickness of $2 \mathrm{~mm}$, and rotation feed of $2 \mathrm{~mm} / \mathrm{s}$. Scans were obtained at the end of inspiration (TLC) from apex to base. Images were reconstructed with a $512 \times 512$ matrix. Parenchymal abnormalities were classified according to the method proposed and validated by Warrick et al. [17]. A point value was assigned to each abnormality, as follows: ground glass appearance $=1$; irregular pleural margins $=$ 2 ; septal and/or subpleural lines $=3$; honeycombing $=4$; subpleural cysts $=5$. A severity score based on the number of abnormalities and an extent score based on the number of pulmonary segments involved were generated. The severity and extent scores were then added to provide a total HRCT score, with a possible range from 0 to 30 . 


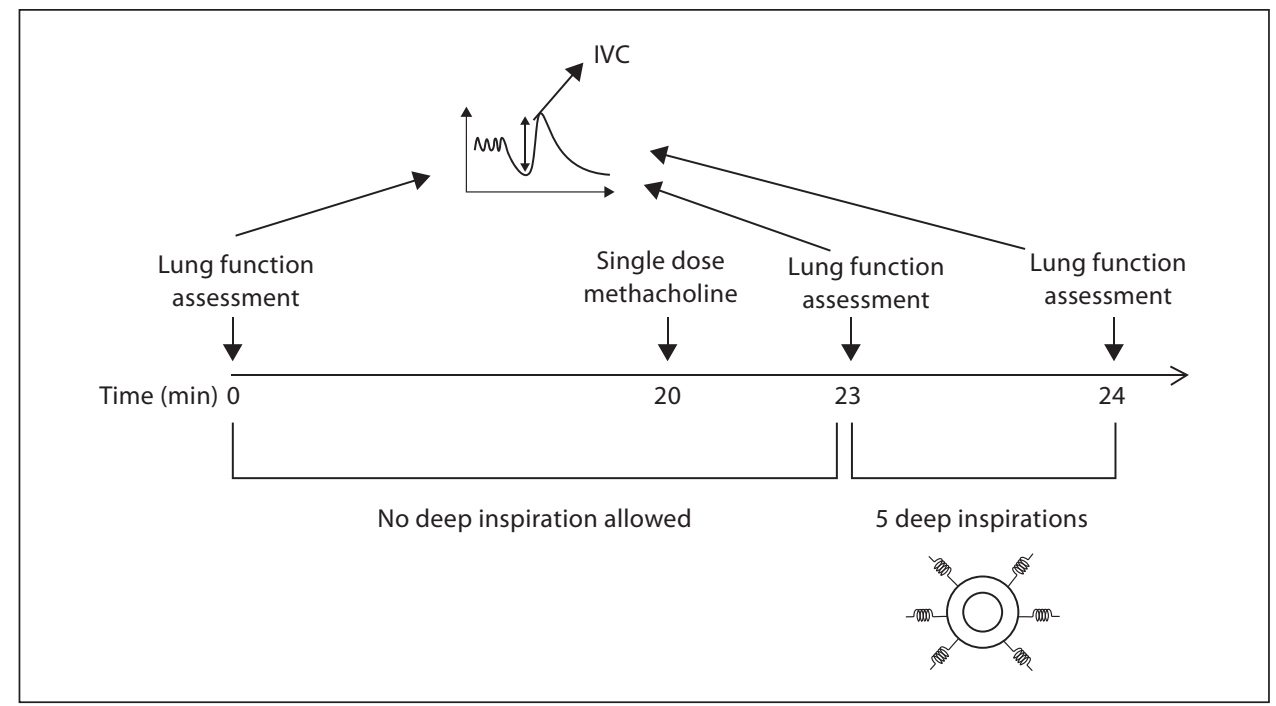

Fig. 1. Single-dose methacholine bronchoprovocation protocol employed to assess the beneficial effect of deep inspirations. The spirometric measurement performed to assess lung function is illustrated in a time-volume curve. This is a combined maneuver (a partial followed by a maximal forced expiratory maneuver), which allows for the IVC to be determined. The singledose methacholine was previously established for each subject by a series of no-deep inspiration bronchoprovocations that were carried out, on separate occasions, with doubling concentrations of methacholine until the dose resulting in the expected reduction in IVC was attained. Subjects were instructed to take deep inspirations $3 \mathrm{~min}$ after inhaling methacholine. In a healthy condition, deep inspirations will pull the airways outward because of the forces of interdependence between the airway wall and the surrounding parenchyma (depicted as springs).

\section{Assessment of the Effect of Deep Inspiration}

Single-dose methacholine bronchoprovocations were performed to induce a reduction of at least $15 \%$ in inspiratory vital capacity (IVC) from baseline, under deep breath prohibition (fig. 1). These bronchoprovocations were repeated, on separate occasions, with doubling concentrations of methacholine until the dose resulting in the expected reduction in IVC was attained, or the maximum concentration of methacholine $(75 \mathrm{mg} / \mathrm{ml})$ was delivered. To measure IVC, the subject was asked to perform a partial forced expiratory maneuver (from the end of a tidal inspiration to residual volume, RV) immediately followed by a maximal forced expiratory maneuver. This combination (partial followed by maximal) maneuver also allowed us to record conventional spirometric outcomes such as $\mathrm{FEV}_{1}$ and FVC. Because lung function measurements in this protocol should be conducted in the absence of deep inspirations, the approach we have repeatedly taken in these types of studies is to use IVC as the primary outcome. IVC has the advantage over $\mathrm{FEV}_{1}$ or FVC of not being affected by a preceding deep inspiratory maneuver.

At baseline, the best of 3 acceptable combination maneuvers was retained. Subjects were then instructed to abstain from taking deep breaths for a period of $20 \mathrm{~min}$. Thereafter, a single concentration of methacholine (Lofarma, Milano, Italy) was administered with the use of a dosimeter (Mefar Elettromedicali, Bovezzo, Italy) as 5 tidal breaths followed, 3 min later, by a single combination spirometric measurement, as described above.
In the subgroup of subjects in whom the targeted reduction in IVC from baseline was reached, the protocol was extended with 4 deep inspirations performed immediately after the postmethacholine combination maneuver. For each deep inspiration, subjects were instructed to inhale slowly through the nose and, without breath holding at TLC, to passively exhale to functional residual capacity (FRC). Following these deep inspirations, another single combination spirometric maneuver was performed. To assess the effect of the deep inspiratory maneuvers, the change in lung function from baseline recorded with the first postmethacholine spirometry was compared with that recorded after the 4 deep inspirations, and expressed as percent effect of deep inspiration, both for IVC and $\mathrm{FEV}_{1}$.

\section{Data Analysis}

Data are reported as means \pm SEM. Simple regression analyses were performed to correlate the effect of deep inspirations, or the percent reduction in IVC in the absence of deep inspirations, with DLCO $\%$ predicted, HRCT score, $\mathrm{FEV}_{1} / \mathrm{FVC}$, TLC $\%$ predicted, RV\% predicted and FRC\% predicted. Unpaired t tests were used to compare the 2 study groups (ISSc vs. dSSc) in terms of demographic and functional variables. In all analyses, 2 -tailed values of $\mathrm{p} \leq 0.05$ were considered statistically significant. 
Table 1. Demographic and functional characteristics of all study subjects

$\begin{array}{lc}\text { Male/female } & 1 / 30 \\ \text { Age, years } & 52 \pm 2.0 \\ \mathrm{FEV}_{1} / \mathrm{FVC} & 0.81 \pm 0.01 \\ \mathrm{FEV}_{1}, \% \text { predicted } & 89 \pm 3.1 \\ \text { FRC, \% predicted } & 85 \pm 3.6 \\ \mathrm{RV}, \% \text { predicted } & 85 \pm 4.0 \\ \mathrm{TLC}, \% \text { predicted } & 86 \pm 3.1 \\ \text { DLCO, \% predicted } & 63 \pm 3.7 \\ \text { HRCT score } & 10 \pm 1.3 \\ \text { PAP, mm Hg } & 33 \pm 1.9\end{array}$

\section{Results}

Sixteen subjects were defined as having diffuse scleroderma and 15 subjects had the limited form of the disease. Time from diagnosis of SSc to inclusion in the studies ranged between 5 months and 21 years. Demographics and baseline lung function characteristics for all subjects are summarized in table 1 . DLCO\% predicted was inversely associated with the HRCT score $(\mathrm{r}=-0.44, \mathrm{p}=$ 0.03 ). The mean pulmonary arterial pressure was significantly correlated with DLCO $\%$ predicted $(\mathrm{r}=-0.55, \mathrm{p}=$ $0.003)$, but not with the HRCT score $(r=0.08, p=0.72)$.

By protocol design, in order to calculate the effect of deep inspirations, we selected only those subjects who attained a reduction of 15\% or higher in IVC from baseline, in the absence of deep inspirations, after methacholine inhalation. A total of 19 subjects were eligible for this part of the study. Ten of them had 1SSc and 9 dSSc. DLCO\% predicted ranged from 22 to $100 \%$ and the HRCT scores from 5 to 14 . The DLCO\% predicted, but not the HRCT score, correlated with the degree of pulmonary hypertension, as expressed by the mean pulmonary arterial pressure $(\mathrm{r}=0.48, \mathrm{p}=0.04$ and $\mathrm{r}=0.20, \mathrm{p}=0.45$, respectively); in addition, DLCO $\%$ predicted and HRCT score were not statistically associated $(\mathrm{r}=0.27, \mathrm{p}=0.32)$. TLC\% predicted ranged from 58 to $114 \%$ and was associated with DLCO $\%$ predicted $(\mathrm{r}=0.47, \mathrm{p}=0.05)$, whereas its relationship with the HRCT score did not reach significance $(\mathrm{r}=0.49, \mathrm{p}=0.06)$. Comparison of the 2 clinical groups (dSSc vs. 1SSc) showed that subjects with the diffuse form of scleroderma had a greater degree of pulmonary involvement, when this was expressed by TLC\% predicted (dSSc vs. $1 S S c: 82 \pm 4.4$ vs. $96 \pm 3.9 \%, p=0.03$ ), but not by DLCO $\%$ predicted (dSSc vs. 1 SSc: $57 \pm 6.6$ vs. $73 \pm$ $6.0 \%, \mathrm{p}=0.10)$ nor HRCT score (dSSc vs. ISSc: $11 \pm 1.1$ vs. $9 \pm 0.6 \%, p=0.09)$.
In the 19 subjects whose IVC was reduced by at least $15 \%$ from baseline, the reduction in IVC was $24 \pm 1.8 \%$, and the methacholine dose required to induce this change was $52 \pm 6.6 \mathrm{mg} / \mathrm{ml}$. The effect of deep inspiration in reversing the methacholine-induced reduction in IVC was $48 \pm 8.4 \%$. For $\mathrm{FEV}_{1}$, the reduction from baseline was $19 \pm 2.0 \%$ and the reversing effect of deep inspiration was $26 \pm 9.2 \%$. The effect of deep inspiration did not differ between the 2 clinical groups (dSSc vs. 1SSc: $51 \pm$ 14.7 vs. $44 \pm 9.9 \%, \mathrm{p}=0.69$ ).

We performed simple regression analyses to evaluate whether lung alterations, as assessed by DLCO\% predicted and the HRCT score, influence the ability of deep inspirations to improve lung function in the constricted airways. The effect of deep inspirations decreased with decreasing DLCO $\%$ predicted ( $r=0.55, p=0.01$; fig. 2 ). The relationship remained significant $(r=0.41, \mathrm{p}=0.05)$ even when the only subject with apparent worsening after deep inspiration (effect of deep inspiration: -40\%) was excluded from the analysis. In the subjects with less than $70 \%$ DLCO $(n=12)$, the average bronchodilation achieved by deep inspirations was $35 \pm 11 \%$, whereas in the subjects with DLCO $>70 \%(n=7)$, the average bronchodilation by deep inspirations was $68 \pm 10 \%$, a value similar to that observed in healthy individuals in other studies $[3,14]$. The observation with IVC was confirmed when $\mathrm{FEV}_{1}$ was employed to calculate the beneficial effect of deep inspiration $(\mathrm{r}=0.54, \mathrm{p}=0.02)$. The HRCT score, on the other hand, was not associated with the effect of deep inspiration $(r=0.22, p=0.42)$. The lack of a relationship between the effect of deep inspiration and imaging abnormalities of the lung was confirmed when the HRCT score was partitioned into a severity HRCT score $(\mathrm{r}=$ $0.12, p=0.65)$ and an extension of lesions HRCT score $(\mathrm{r}=0.29, \mathrm{p}=0.27)$. To explore whether the magnitude of lung restriction, as measured by lung function, could be responsible for the reduction in the effect of deep inspiration, we conducted simple regression analyses between the percent effect of deep inspiration and various baseline parameters. We found no significant correlation with TLC $\%$ predicted $(\mathrm{r}=0.39, \mathrm{p}=0.11), \mathrm{RV} \%$ predicted $(\mathrm{r}=$ $0.13, \mathrm{p}=0.60)$ or FRC $\%$ predicted $(\mathrm{r}=0.41, \mathrm{p}=0.09)$.

An interesting observation was also made: in the absence of deep inspiration, in 12 of the 31 study subjects (38\%), even when the highest concentration of methacholine $(75 \mathrm{mg} / \mathrm{ml})$ was delivered, IVC did not decrease by $\geq 15 \%$. In previous work that our group has conducted on healthy individuals using an identical provocation technique, only $20 \%$ did not reach the targeted reduction in IVC with the same doses of methacholine [18]. These 


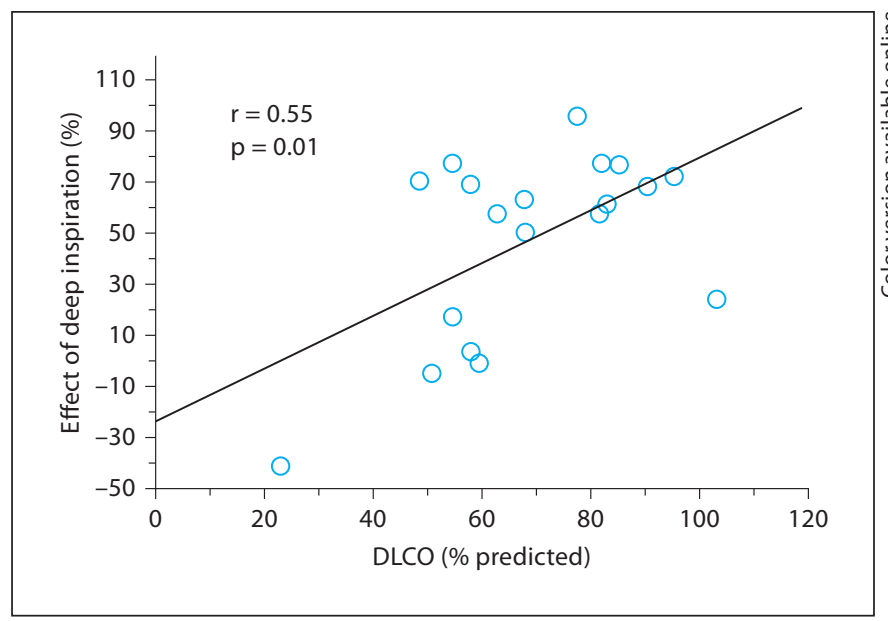

Fig. 2. Correlation between the effect of deep inspiration and DLCO.

data suggested that, in scleroderma, the direct response of the airways to the bronchoconstrictor is reduced. It became, therefore, interesting to explore the factors that influence responsiveness to methacholine, in the absence of deep inspirations, in our population of scleroderma subjects. In order to quantify responsiveness, we divided the percent reduction in IVC by the methacholine dose employed in the no-deep inspiration bronchoprovocation (\% IVC reduction/single-dose methacholine). We have previously employed this outcome (reactivity index) in healthy individuals [18] to describe airway responsiveness to single-dose methacholine challenges, even when the desired target for lung function reduction has not been met. The reactivity index in our entire scleroderma population was $0.54 \pm 0.12$. Figure 3 shows the correlation of the reactivity index with DLCO\% predicted $(\mathrm{r}=$ $0.51, p=0.003)$ and suggests that, in the absence of deep inspirations, functional abnormalities of scleroderma lung protect against the reduction in IVC by methacholine. This is also true when the reactivity index is calculated by using $\mathrm{FEV}_{1}$ instead of IVC $(\mathrm{r}=0.37, \mathrm{p}=0.04)$. In fact, we saw minimal responsiveness in all subjects whose DLCO was $<50 \%$ predicted (reactivity index for DLCO $<50 \%$ : $0.15 \pm$ 0.05; DLCO > 50\%: $0.70 \pm 0.16, \mathrm{p}=0.04$ ). We did not find a significant correlation between the HRCT score and the reactivity index $(\mathrm{r}=0.32, \mathrm{p}=0.14)$, or between the reactivity index and baseline lung function outcomes indicative of restriction (for TLC\% predicted: $\mathrm{r}=0.20, \mathrm{p}=0.29$; for $\mathrm{RV} \%$ predicted: $\mathrm{r}=0.05, \mathrm{p}=$

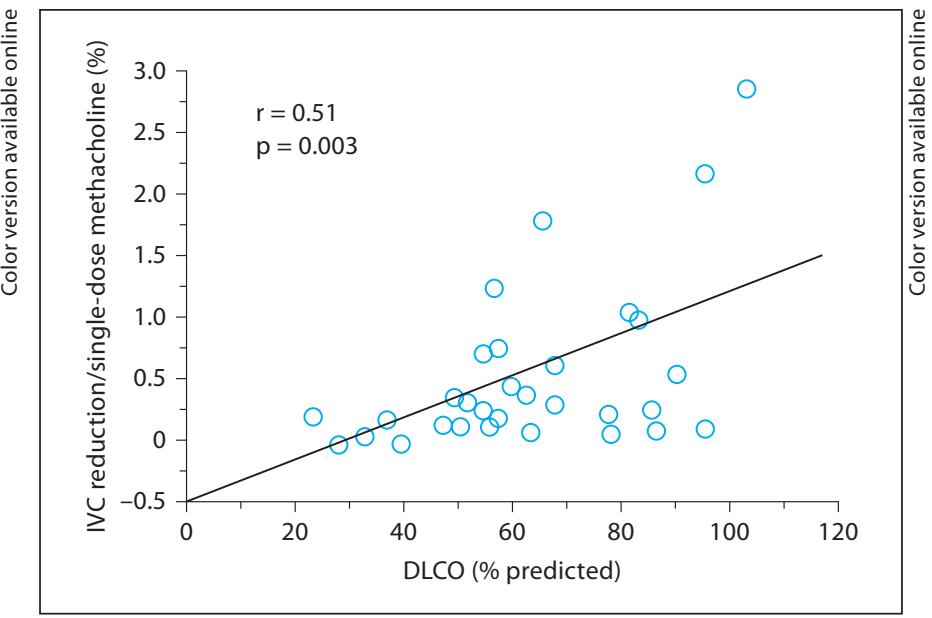

Fig. 3. Correlation between the reactivity index and DLCO. The reactivity index is the ratio of the percent reduction in IVC from baseline in the no-deep inspiration bronchoprovocation over the single-dose methacholine employed in the protocol.

0.98; for FRC\% predicted: $r=0.01, p=0.96$ ). Finally, when we compared those subjects who reacted to methacholine with $>15 \%$ reduction in IVC versus those who did not, we found no differences in their baseline airflow parameters ( $\mathrm{FEV}_{1} \%$ predicted: $84 \pm 5.3$ vs. $92 \pm 3.7 \%, \mathrm{p}=0.20$; $\mathrm{FEV}_{1} / \mathrm{FVC}: 0.82 \pm 0.02$ vs. $0.81 \pm 0.02, \mathrm{p}=0.90$; for reactors vs. nonreactors).

\section{Discussion}

In this study, our goal was to test the hypothesis in the absence of deep inspirations, that the beneficial effect of deep inspirations decreases with increasing magnitude of scleroderma lung involvement, as determined by DLCO $\%$ predicted. We also made the observation that, in those individuals with the lowest DLCO\% predicted, the airway response to methacholine, in the absence of deep inspirations, was reduced, compared to historic healthy controls. As a result, it was only possible to assess the effect of deep inspiration on subjects who, in their majority, had DLCO $\geq 50 \%$ predicted (fig. 2 ).

SSc is a multisystem connective tissue disorder characterized by widespread micro- and macrovascular damage, and by fibrosis of the skin and internal organs. Lung involvement is a frequent manifestation in patients with SSc, and is the leading cause of morbidity and mortality [19]. Lung involvement can manifest itself by interstitial lung disease, which occurs in approximately $40 \%$ of pa- 
tients, and/or by pulmonary hypertension, occurring in up to $50 \%$ of patients [20]. The wide range of pulmonary involvement was demonstrated in this study by the spectrum of DLCO\% predicted values and HRCT abnormalities. The reduction in DLCO is considered a sensitive marker of lung involvement, in that it precedes the restrictive pattern characterized by reduction in lung volumes [21]. However, diffusion across the alveolar capillary units may be altered by factors other than interstitial fibrosis. In subjects with scleroderma, the reduction in DLCO could be attributed to alterations in the vascular compartment. This is supported by the significant relationship between the diffusing capacity and the degree of pulmonary hypertension, as demonstrated by other investigators [22], and confirmed in our subjects. HRCT abnormalities correlate with lung histology [23] and reflect the fibrotic process of the lung. The HRCT score that was applied in our study population has been validated in subjects with scleroderma: a minimum score of 7 has been proposed as predictive of lung impairment [24]. Only 3 subjects had an HRCT score lower than 7, indicating that lung abnormalities were present even in asymptomatic subjects and confirming that the population that we tested fulfilled the necessary criteria for interstitial lung disease.

We have recently demonstrated that the beneficial effect of deep inspiration is reduced in subjects with COPD [8], and that this impairment is related to the percentage of destroyed alveolar attachments in the lung [9]. This supports the hypothesis that deep inspiration exerts its effects by distending the airways and that this distension is mediated through the elastic forces of the connective tissue of the lung. The same forces account for the largest component of the elastic recoil of the lung, which is greatly diminished in emphysema. It was, therefore, reasonable to propose that the beneficial effect of deep inspirations would be enhanced in conditions in which the elastic recoil forces are increased, such as that of lung fibrosis $[25,26]$. However, our data not only do not support this hypothesis, but indicate that the effects of deep inspiration diminish with increasing severity of scleroderma lung, as measured by the diffusion capacity.

A clear limitation of our study is the lack of elastic recoil measurements in our scleroderma subjects. Such measurements would have allowed us to test in a more definitive way whether a relationship between elastic recoil and the beneficial effects of deep inspiration exists, even if it was inverse to the one we had predicted. However, it has been previously demonstrated that a close relationship between elastic recoil and diffusion or imaging abnormalities exists [27-29]. Another limitation we need to point out is that the majority of our subjects (26 of 31) were receiving calcium channel blockers at the time of evaluation. Although a body of literature suggests that these medications could affect airway smooth muscle contractility, the data are conflicting [30, 31]. Furthermore, examination of the results from the 5 subjects who were not on calcium channel blockers did not reveal overt differences from the subjects who were using these medications neither in airway responsiveness to methacholine in the absence of deep inspirations, nor in the effects of deep inspiration.

In retrospect, various factors can be considered to explain the reduced ability of deep inspirations to reverse the changes induced by methacholine. The contribution of lung restriction or of fibrotic chest wall changes can be reasonably ruled out, since baseline lung volume outcomes, including TLC\% predicted, did not influence the effectiveness of deep inspiration. The next possibility is that lung inflation through deep inspiration may have been unable to stretch the airways in order to reverse the effects of methacholine. Our study did not assess this possibility, since HRCT was not performed both at low and high lung volumes. Theoretically, airway distension may have been diminished because of stiffened airways. In connective tissue disorders, the vascular compartment undergoes structural changes that are characterized by intimal hyperplasia and fibrosis of the vessel media and adventitia [32]. In the lungs, the resulting pulmonary hypertension involves not only vascular, but also bronchial smooth muscle hypertrophy, and the bronchi appear to have undergone remodeling changes towards fibrosis [33]. Airway stiffness may not necessarily reflect fibrosis. Pulmonary hypertension could lead to peribronchial engorgement that causes external compression of the airways and opposes airway distension. This could explain the discrepancy between DLCO and HRCT in their relationships with the bronchodilatory effect of deep inspiration: it is possible that the component of the diffusing capacity that is affected by the vasculature is the major driving factor in the relationship between DLCO and the effect of deep inspiration. This component is not reflected in HRCT imaging.

Compared to historic controls [18], an unusually high number of subjects with scleroderma failed to react to methacholine, even in the absence of deep inspirations. This is an interesting incidental finding as we have not seen it in asthma and COPD. The mechanism of hyporesponsiveness to methacholine is unknown, but its elucidation may be of value in understanding the factors that 


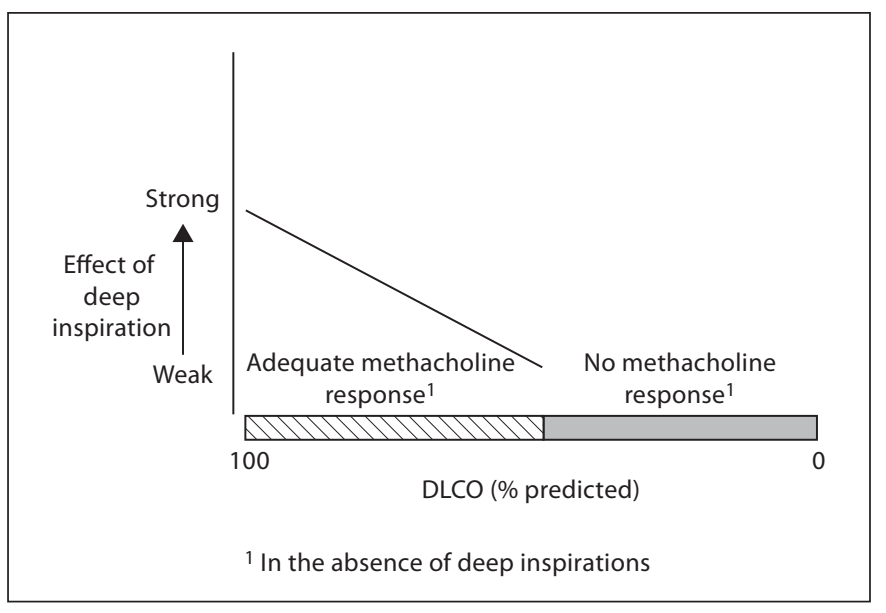

Fig. 4. Schematic conceptualization of the findings of this study. In individuals with SSc and relatively mild diffusing capacitylimiting pulmonary abnormalities, inhaled methacholine can result in bronchoconstriction, but in the same individuals the ability of deep inspirations to reverse the methacholine effects is limited with worsening diffusion capacity. In individuals with even lower DLCO values, methacholine fails to generate bronchoconstriction; in these individuals, the effects of deep inspiration cannot be assessed.

contribute to airway obstructive responses to spasmogens.

Factors that could be responsible for this observation include reduced airway smooth muscle contractility, altered deposition of methacholine or rapid degradation or clearance of methacholine from its sites of action. In addition, the possibility exists that airway wall stiffness or increased baseline airway caliber because of higher pa- renchymal traction forces could diminish the ability of contracted airway smooth muscle to produce early airway closure and airflow limitation. Although we have no information on wall stiffness, we did not find any relationship between reactivity to methacholine in the absence of deep inspirations and baseline airflow parameters. The only predictor of reduced responsiveness to methacholine that we identified was very low DLCO (fig. 3). Of the 12 subjects who did not respond to methacholine, $6(50 \%)$ had DLCO $\leq 50 \%$, whereas of the 19 subjects who reacted to methacholine, only $3(16 \%)$ had DLCO $\leq 50 \%$ predicted.

To summarize our findings (fig. 4), in individuals with SSc and relatively mild diffusing capacity-limiting pulmonary abnormalities, inhaled methacholine in the absence of deep inspirations results in bronchoconstriction, but the ability of deep inspirations to reverse the methacholine effects is limited as a function of worsening diffusion capacity. Our speculation is that this observation reflects the effects of airway wall functional and structural alterations induced by scleroderma lung, but our study does not allow us to identify a specific mechanism. In individuals with more severe pulmonary disease, reflected in even lower DLCO values, the effects of deep inspiration cannot be evaluated because methacholine fails to elicit a bronchoconstrictive response. Perhaps, the same alterations that, in their milder form, impair the beneficial effects of deep inspirations, when more severe, prevent airway smooth muscle constriction from causing airway narrowing. Future work with this model will hopefully improve our understanding of lung and airway function in fibrotic lung disease.

\section{References}

1 Wheatley JR, Pare PD, Engel LA: Reversibility of induced bronchoconstriction by deep inspiration in asthmatic and normal subjects. Eur Respir J 1989;2:331-339.

- 2 Fairshter RD: Effect of a deep inspiration on expiratory flow in normals and patients with chronic obstructive pulmonary disease. Bull Eur Physiopathol Respir 1986;22:119-125.

-3 Scichilone N, Kapsali T, Permutt S, Togias A: Deep inspiration-induced bronchoprotection is stronger than bronchodilation. Am J Respir Crit Care Med 2000;162:910-916.

$\checkmark 4$ Brusasco V, Crimi E, Barisione G, Spanevello A, Rodarte J, Pellegrino R: Airway responsiveness to methacholine: effects of deep inhalations and airway inflammation. J Appl Physiol 1999;87:567-573. $\checkmark 5$ Jensen A, Atileh H, Suki B, Ingenito E, Lutchen K: Selected contribution: airway caliber in healthy and asthmatic subjects: effects of bronchial challenge and deep inspirations. J Appl Physiol 2001;91:506-515.

6 Sundblad BM, Larsson K: Effect of deep inhalations after a bronchial methacholine provocation in asthmatic and non-asthmatic subjects. Respir Med 2002;96:477-481.

7 Scichilone N, Permutt S, Bellia V, Togias A: Inhaled corticosteroids and the beneficial effect of deep inspiration in asthma. Am J Respir Crit Care Med 2005;172:693-698.
8 Scichilone N, Marchese R, Catalano F, Vignola AM, Togias A, Bellia V: Bronchodilatory effect of deep inspiration is absent in subjects with mild COPD. Chest 2004;125: 2029-2035.

-9 Scichilone N, Bruno A, Marchese R, Vignola AM, Togias A, Bellia V: Association between reduced bronchodilatory effect of deep inspiration and loss of alveolar attachments. Respir Res 2005;6:55.

10 Brown RH, Scichilone N, Mudge B, Diemer, FB, Permutt S, Togias A: High-resolution computed tomographic evaluation of airway distensibility and the effects of lung inflation on airway caliber in healthy subjects and individuals with asthma. Am J Respir Crit Care Med 2001;163:994-1001. 
11 Pyrgos G, Scichilone N, Mintz M, Permutt S, Togias A, Brown R: Deep inspiration-induced bronchodilation in asthma is dependent on airways distensibility assessed by HRCT. Am J Respir Crit Care Med 2001;163: A830.

-12 Lim TK, Pride NB, Ingram RH Jr: Effects of volume history during spontaneous and acutely induced air-flow obstruction in asthma. Am Rev Respir Dis 1987;135:591-596.

$\checkmark 13$ Verbeken EK, Cauberghs M, Mertens I, Clement J, Lauweryns JM, Van de Woestijne KP: The senile lung. Comparison with normal and emphysematous lungs. 1. Structural aspects. Chest 1992;101:793-799.

-14 Scichilone N, Marchese R, Catalano F, Togias A, Vignola AM, Bellia V: The bronchodilatory effect of deep inspiration diminishes with aging. Respir Med 2004;98:838-843.

-15 Preliminary criteria for the classification of systemic sclerosis (scleroderma). Subcommittee for scleroderma criteria of the American Rheumatism Association Diagnostic and Therapeutic Criteria Committee. Arthritis Rheum 1980;23:581-590.

-16 LeRoy EC, Black C, Fleischmajer R, et al: Scleroderma (systemic sclerosis): classification, subsets and pathogenesis. J Rheumatol 1988;15:202-205.

-17 Warrick JH, Bhalla M, Schabel SI, Silver RM: High resolution computed tomography in early scleroderma lung disease. J Rheumatol 1991;18:1520-1528.
8 Scichilone N, Morici G, Marchese R, et al: Reduced airway responsiveness in nonelite runners. Med Sci Sports Exerc 2005;37: 2019-2025.

19 Lee P, Langevitz P, Alderdice CA, et al: Mortality in systemic sclerosis (scleroderma). Q J Med 1992;82:139-148.

20 Hachulla E, Gressin V, Guillevin L, et al: Early detection of pulmonary arterial hypertension in systemic sclerosis: a French nationwide prospective multicenter study. Arthritis Rheum 2005;52:3792-3800.

21 Peters-Golden M, Wise RA, Hochberg MC, Stevens MB, Wigley FM: Carbon monoxide diffusing capacity as predictor of outcome in systemic sclerosis. Am J Med 1984;77:10271034.

22 Lettieri CJ, Nathan SD, Barnett SD, Ahmad S, Shorr AF: Prevalence and outcomes of pulmonary arterial hypertension in advanced idiopathic pulmonary fibrosis. Chest 2006; 129:746-752.

23 Wells AU, Hansell DM, Corrin B, et al: High resolution computed tomography as a predictor of lung histology in systemic sclerosis. Thorax 1992;47:738-742.

24 Diot E, Boissinot E, Asquier E, et al: Relationship between abnormalities on high-resolution CT and pulmonary function in systemic sclerosis. Chest 1998;114:1623-1629.

25 Blom-Bulow B, Jonson B, Brauer K: Lung function in progressive systemic sclerosis is dominated by poorly compliant lungs and stiff airways. Eur J Respir Dis 1985;66:1-8.
26 Chetta A, Marangio E, Olivieri D: Pulmonary function testing in interstitial lung diseases. Respiration 2004;71:209-213.

$>27$ Scheja A, Akesson A, Wollmer P, Wollmer FA: Early pulmonary disease in systemic sclerosis: a comparison between carbon monoxide transfer factor and static lung compliance. Ann Rheum Dis 1993;52:725729.

28 Sud A, Gupta D, Wanchu A, Jindal SK, Bambery P: Static lung compliance as an index of early pulmonary disease in systemic sclerosis. Clin Rheumatol 2001;20:177-180.

29 Pistelli R, Maini CL, Fuso L, et al: Pulmonary involvement in progressive systemic sclerosis. Respiration 1987;51:296-306.

30 Moscato G. Danna P, Dorigo N, Dellabianca A, Candura F: Effect of nifedipine on hyperreactive bronchial responses to methacholine. Ann Allergy 1986;56:145-149.

31 Matthews JI, et al: Nifedipine does not alter methacholine-induced bronchial reactivity. Ann Allergy 1984;53:462-467.

32 Denton CP, Black CM, Abraham DJ: Mechanism and consequences of fibrosis in systemic sclerosis. Nat Clin Pract Rheumatol 2006; 2:134-144.

33 Schindler MB, Bohn DJ, Bryan AC, Cutz E, Rabinovitch M: Increased respiratory system resistance and bronchial smooth muscle hypertrophy in children with acute postoperative pulmonary hypertension. Am J Respir Crit Care Med 1995;152:1347-1352. 